\title{
Hydrodynamic analysis of wave and cylinder fish cage interactions
}

\author{
M. Ma ${ }^{1}$, H. Zhang ${ }^{2}$ and D. Jeng ${ }^{2}$ \\ ${ }^{1} S c h o o l$ of Engineering and Built Environment \\ Griffith University, Gold Coast QLD 4222, Australia \\ ${ }^{2}$ School of Engineering and Built Environment \\ Griffith University, Gold Coast QLD 4222, Australia
}

\begin{abstract}
Submerged net cages have been commonly used for offshore fish farming. The present study analyses the linear elastic behaviour of a cylindrical fish cage interaction with linear waves, based on the potential flow theory. The fishnet is treated as a porous membrane, therefore, Darcy's law is applied to deal with the pressure-velocity boundary conditions on the net interface. The flow field is divided into two regions where the scattered velocity potentials are solved by the Fourier-Bessel series and the least square approximation methods. Numerical results show that the higher amplitude of dynamic pressure difference acting on the net surface due to the wave is mainly concentrated on the upper part of the fish cage. The present study provides a feasible prediction of the wave action estimations and the dynamic response of the net cage structure, which are key factors for the design of the offshore fish cage.
\end{abstract}

\section{Keywords}

Fish cage; Porous membrane; Potential flow; Fourier-Bessel expansion; Hydro-elastic analysis; Least square method.

\section{Introduction}

Cylinder net cages are commonly used for nearshore fish farming, to utilise ocean water resources. However, due to nearshore pollution increases, the feasibility of installing cages in the deep sea has gradually attracted attention from the fishing industry. Offshore fish farming is beneficial to change the current situation of nearshore aquaculture and the environmental deterioration along the coast and the inner bay. Although deep-sea fish cages have been used recently, there are still difficulties and challenges in the engineering design. Compared with shallow water, the sea conditions in the deep sea are more complicated and the environmental loads are more intense. In extreme cases, the destruction and collapse of floating fish cages cause the fish to escape or die, causing significant economic losses. Therefore, the hydrodynamic analysis is essential to evaluate the mechanical performance and reliability of the net cages.

Some researchers have conducted a series of studies on the net cage and similar structures. At present, methods used for the prediction of the hydrodynamics in the vicinity of the net structure include empirical approaches, potential flow models, and computational fluid dynamics (CFD) models. The empirical formula mainly evaluates the fluid forces acting on the net through the Morrison equations [8] and screen type model [5], where the net is modelled as the composition of twines and knots. However, the interference of the net structure to the wave field is not considered for the empirical approaches. The potential flow models obtain the corresponding incident wave potential and scatter wave potential by linear superposition solving the Laplace's equation [2], but the fluid is assumed to be inviscid and irrotational. The CFD models consider the net as a porous medium zone and solve the NavierStokes equation to obtain the flow field [3]. The CFD models usually require relatively fine grid scales, so high computational costs are inevitable for large domain simulations.

The present study will focus on the hydrodynamic analysis of wave interaction with a large-scale cylindrical fish cage in deep water. A semi-analytical method based on the potential flow theory is used to describe the flow field. The small-amplitude wave theory is applied for the wave field. The fishnet is treated as a flexible porous medium membrane. Then, the deformation of the cylinder net is expressed by using the one-dimensional string vibration equation. The flow velocity-pressure relationship on the net surface is governed by Darcy's law. The solution of the velocity potential is expressed in the form of Fourier-Bessel expansion, which is solved by matching boundary conditions and the least square method.

\section{Research method}

Governing equations and boundary conditions

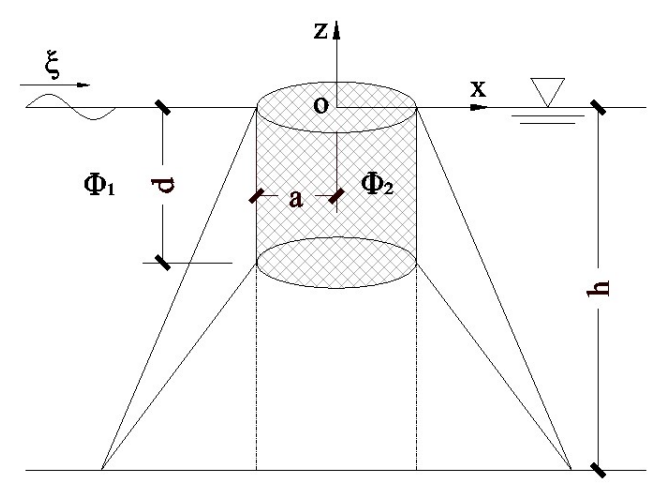

Figure 1. Flow field and cage sketch

In this study, a cylinder net cage was considered, where its upper and lower ends are assumed to be fixed as shown in figure 1 . The $z$-axis of the cylindrical coordinate system $(r, \theta, z)$ is at the centre of the cylindrical cage. The mean water level is at $z$ $=0$. The cage has a radius of $r=a$ and a height of $d$, in the water with a finite water depth of $h$. The flow field is divided into two zones, where Zone $1(r>a,-h<z<0)$ is in the external region outside the net cage and Zone $2(r>a,-h<z<0)$ is in the inner region of the net cage. In addition, $S_{\text {net }}$ is the net portion $(-d \leqslant$ $z \leqslant 0)$, and $S_{\text {gap }}$ is the gap region $(-h \leqslant z<-d)$. The fluid is assumed to be irrotational and incompressible with density $\rho$, 
and the viscosity is neglected. $\Phi_{1}$ and $\Phi_{2}$ represent the velocity potential in Zone 1 and 2, respectively. Considering a linear wave action with an angular frequency $\omega$ and a wave height $H$, the velocity potential $\Phi(r, \theta, z, t)$ of the flow field satisfies:

$$
\Phi_{j}=R_{e}\left[\phi_{j}(r, \theta, z)\right] e^{-i \omega t}, j=1,2
$$

where, $\phi_{j}$ is the complex velocity potential amplitude, and satisfies the Laplace's Equation:

$$
\frac{\partial^{2} \phi_{j}}{\partial r^{2}}+\frac{1}{r} \frac{\partial \phi_{j}}{\partial r}+\frac{1}{r^{2}} \frac{\partial^{2} \phi_{j}}{\partial \theta^{2}}+\frac{\partial^{2} \phi_{j}}{\partial z^{2}}=0, j=1,2
$$

The velocity potential amplitude $\phi$ consists of two components, i.e., incident wave velocity potential $\phi^{I}$, and the scatter wave velocity potential $\phi^{S}$ due to diffraction and radiation:

$$
\phi=\phi^{I}+\phi^{s}
$$

For the free water surface, it satisfies that:

$$
\frac{\partial \phi_{j}}{\partial z}-\frac{\omega^{2}}{g} \phi_{j}=0, z=0 \text { and } j=1,2
$$

The slip boundary condition is applied on the seabed:

$$
\frac{\partial \phi_{j}}{\partial z}=0, z=-h \text { and } j=1,2
$$

When $r$ approaches infinity, the scattered wave potential $\phi^{s}$ meets the Sommerfeld condition:

$$
\lim _{r \rightarrow \infty} \sqrt{r}\left(\frac{\partial \phi^{s}}{\partial r}-i k_{0} \phi^{s}\right)=0
$$

where $k_{0}$ is the incident wavenumber.

On the net surface $(r=a)$, the flow passes through the porous membrane satisfying Darcy's law [4]:

$$
\begin{gathered}
\frac{\partial \phi_{j}}{\partial r}=i k_{0} G\left(\phi_{2}-\phi_{1}\right)+i \omega \eta \cos \theta \\
r=a, z \in S_{\text {net }}, j=1,2
\end{gathered}
$$

where $\eta$ is the complex horizontal displacement amplitude of the net cage cylinder; $G$ is the porous-effect parameter, which is given by Chwang [4],

$$
G=G_{r}+i G_{i}=\frac{\delta(f+i S)}{k_{0} d_{s}\left(f^{2}+S^{2}\right)}
$$

in which: $\delta$ is porous ratio; $d_{s}$ is the porous medium thickness; $f$ is the resistance coefficient, and $S$ is the inertial force coefficient. The real part $G_{r}$ indicates the resistance effect of the porous media, and the imaginary part $G_{i}$ represents the fluid inertia effect in porous media. The parameters $f$ and $S$ may be empirically evaluated following Madsen [6].

Also, the continuity of normal velocity satisfies:

$$
\begin{gathered}
\frac{\partial \phi_{1}}{\partial r}=\frac{\partial \phi_{2}}{\partial r}, r=a, z \in S_{n e t} \\
\phi_{1}=\phi_{2}, r=a, z \in S_{\text {gap }}
\end{gathered}
$$

The horizontal deflection of the porous membrane is assumed $\zeta=\operatorname{Re}\left[\eta(z) e^{-i \omega t}\right]$, and the complex displacement amplitude $\eta$ satisfies the linearised dynamic condition proposed by AbulAzam and Williams [1]:

$$
\begin{gathered}
\gamma \frac{d^{4} \eta}{d z^{4}}+\frac{\beta}{h^{2}} \frac{d^{2} \eta}{d z^{2}}-\alpha \eta= \\
\frac{2 a i}{\tau g h^{5}} \int_{0}^{\pi}\left(\phi_{1}-\phi_{2}\right) \cos (\pi-\theta) d \theta
\end{gathered}
$$

where: $\gamma=E I /\left(m_{s} g h^{3}\right)$ is the non-dimensional stiffness of the net cage; $E I$ is the flexural stiffness of the net cage; $\beta=$ $Q /\left(m_{s} g h\right)$ is the non-dimensional compressive force acting on the net cage; $\alpha=\omega^{2} /\left(g h^{3}\right) ; \tau=m_{s} /\left(\rho h^{2}\right) ; m_{s}$ is the uniform mass per unit length, and $\rho$ is the water density.
For the fixed edge of the net cage, the deflection and slope are zero, therefore:

$$
\eta=0, \frac{d \eta}{d z}=0, r=a, z=0 \text { or }-d
$$

\section{Analytical solutions}

The general expression of $\phi_{1}$, which meets the equation (2), (4), (5), and (6):

$$
\phi_{1}=\sum_{m=0}^{\infty}\left(\phi_{I m}+\phi_{1 m}^{s}\right) \cos (m \theta)
$$

where,

$$
\begin{gathered}
\phi_{I m}=-\frac{i g H}{2 \omega} \frac{\cosh \left[k_{0}(z+h)\right]}{\cosh \left(k_{0} h\right)} \lambda_{m} J_{m}\left(k_{0} r\right) \\
\lambda_{m}=\left\{\begin{array}{cc}
1, m=0 \\
2 i^{m}, m>0
\end{array}\right. \\
\phi_{1 m}^{s}=\sum_{n=0}^{\infty} A_{m n} R_{m}^{1}\left(k_{n} r\right) f_{n}(z) \\
R_{m}^{1}\left(k_{n} r\right)= \begin{cases}\frac{H_{m}\left(k_{n} r\right)}{H_{m}^{\prime}\left(k_{n} a\right)}, & n=0 \\
\frac{K_{m}\left(k_{n} r\right)}{K_{m}^{\prime}\left(k_{n} a\right)}, & n \geq 0\end{cases} \\
f_{n}(z)= \begin{cases}\frac{\cosh \left[k_{n}(z+h)\right]}{\cosh \left(k_{n} h\right)}, & n=0 \\
\frac{\cos \left[k_{n}(z+h)\right]}{\cos \left(k_{n} h\right)}, & n \geq 0\end{cases}
\end{gathered}
$$

Similarly, for region 2 , the velocity potential satisfies the governing equation (2), (4) and (5), therefore the general solutions of $\phi_{2}$ is:

$$
\phi_{2}=\sum_{m=0}^{\infty}\left(\phi_{I m}+\phi_{2 m}^{s}\right) \cos (m \theta)
$$

where,

$$
\begin{gathered}
\phi_{2 m}^{S}=\sum_{n=0}^{\infty} B_{m n} R_{m}^{2}\left(k_{n} r\right) f_{n}(z) \\
R_{m}^{2}\left(k_{n} r\right)= \begin{cases}\frac{J_{m}\left(k_{n} r\right)}{J_{m}^{\prime}\left(k_{n} a\right)}, & n=0 \\
\frac{I_{m}\left(k_{n} r\right)}{I_{m}^{\prime}\left(k_{n} a\right)}, & n \geq 0\end{cases}
\end{gathered}
$$

$k_{n}$ is the positive real root of the following equations:

$$
\left\{\begin{array}{ll}
v=k_{n} \tanh \left(k_{n} h\right), & n=0 \\
v=-k_{n} \tan \left(k_{n} h\right), & n>0
\end{array}, \quad v=\frac{\omega^{2}}{g}\right.
$$

$A_{m n}$ and $B_{m n}$ are unknown coefficients. $J_{m}$ is the first kind of Bessel functions of $\mathrm{m}$ orders, $H_{m}$ is the Hankel function of $\mathrm{m}$ orders, $I_{m}$ is the first kind of modified Bessel function of $\mathrm{m}$ orders, and $K_{m}$ is the second kind of modified Bessel function of $\mathrm{m}$ orders.

Because of the normal velocity continuity, substitute the equation (13) and (19) into the equation (9), then:

$$
A_{m n}=B_{m n}
$$

Therefore, on the net surface, the velocity potential drop has:

$$
\begin{gathered}
\Delta \phi=\phi_{1}-\phi_{2}= \\
\sum_{m=0}^{\infty} \sum_{n=0}^{\infty} A_{m n} X_{m n} f_{n}(z) \cos (m \theta)
\end{gathered}
$$

where:

$$
X_{m n}=R_{m}^{1}\left(k_{n} a\right)-R_{m}^{1}\left(k_{n} a\right)
$$

Substituting equation (24) into equation (11), then equation (11) can be rewritten as:

$$
\begin{gathered}
a_{1} \frac{d^{4} \eta}{d z^{4}}+a_{2} \frac{d^{2} \eta}{d z^{2}}+a_{3} \eta= \\
a_{4} \sum_{n=0}^{\infty} A_{1 n} X_{1 n} f_{n}(z)
\end{gathered}
$$


where, $a_{1}=\gamma, a_{2}=\frac{\beta}{h^{2}}, a_{3}=-\alpha$, and $a_{4}=-\frac{\pi a i \omega}{\tau g h^{5}}$.

Therefore, the general solution of $\eta$ is:

$$
\eta(z)=\sum_{q=1}^{4} C_{q} e^{\kappa_{q} z}+\sum_{n=0}^{\infty} F_{n} A_{1 n} f_{n}(z)
$$

where,

$$
F_{n}= \begin{cases}\frac{a_{4} X_{1 n}}{a_{1} k_{n}^{4}+a_{2} k_{n}^{2}+a_{3}}, & n=0 \\ \frac{a_{4} X_{1 n}}{a_{1} k_{n}^{4}-a_{2} k_{n}^{2}+a_{3}}, & n \geq 1\end{cases}
$$

$\kappa_{q}$ are the roots of:

$$
a_{1} \kappa^{4}+a_{2} \kappa^{2}+a_{3}=0
$$

For the net region, substituting equation (13), (24) and (27) into equation (7), a system of equations can be obtained:

$$
S_{m}(z)=\sum_{n=0}^{\infty} A_{m n} \epsilon_{m n}(z)+\mu_{m}(z)=0
$$

in which, when $m \neq 1$,

$$
\begin{gathered}
\epsilon_{m n}(z)=\left\{\begin{array}{c}
\left(k_{n}+i k_{0} G X_{m n}\right) f_{n}(z), z \in S_{n e t} \\
X_{m n} f_{n}(z), z \in S_{\text {gap }}
\end{array}\right. \\
\mu_{m}(z)=\left\{\begin{array}{c}
-\frac{i g H k_{0}}{2 \omega} \frac{\cosh \left[k_{0}(z+h)\right]}{\cosh \left(k_{0} h\right)} \lambda_{m} J_{m}^{\prime}\left(k_{0} a\right), z \in S_{\text {net }} \\
0, z \in S_{\text {gap }}
\end{array}\right.
\end{gathered}
$$

for $m=1$,

$$
\begin{gathered}
\epsilon_{m n}(z)=\left\{\begin{array}{c}
\left(k_{n}+i k_{0} G X_{m n}-i \omega F_{n}\right) f_{n}(z), \quad z \in S_{n e t} \\
X_{m n} f_{n}(z), \quad z \in S_{g a p}
\end{array}\right. \\
\mu_{m}(z)=\left\{\begin{array}{c}
-\frac{i g H k_{0}}{2 \omega} \frac{\cosh \left[k_{0}(z+h)\right]}{\cosh \left(k_{0} h\right)} \lambda_{m} J_{m}^{\prime}\left(k_{0} a\right) \\
-i \omega \sum_{q=1}^{4} C_{q} e^{\kappa_{q} z}, \quad z \in S_{\text {net }} \\
0, \quad z \in S_{\text {gap }}
\end{array}\right.
\end{gathered}
$$

For equation (30), using the least-squares approximation method yields $[2,7]$ :

$$
\int_{-h}^{0}\left|S_{m}(z)\right|^{2} d z=\min \Rightarrow \int_{-h}^{0} S_{m}^{*} \frac{\partial S(z)}{\partial A_{m n}} d z=0
$$

Substituting equation (30) into equation (35), an equation system of $M \times N \times q$ for $A_{m n}$ is obtained:

$$
\begin{gathered}
\sum_{n=0}^{N} A_{m n}^{*} \Psi_{m n q}=\Omega_{m q}, \\
m=0,1, \ldots, M \text { and } q=0,1, \ldots, N
\end{gathered}
$$

where,

$$
\Psi_{m n q}=\int_{-h}^{0} \epsilon_{m n}^{*} \epsilon_{m q} d z, \Omega_{m q}=-\int_{-h}^{0} \mu_{m}^{*} \epsilon_{m q} d z
$$

Therefore, according to linearised Bernoulli's equation, the dynamic pressure $p$ of the flow field is:

$$
p_{j}=\operatorname{Re}\left[-\rho \frac{\partial\left(\phi_{j} e^{-i \omega t}\right)}{\partial t}\right] \quad j=1,2
$$

The pressure difference acting on the net surface is defined as:

$$
\Delta p=p_{1}-p_{2}, r=a
$$

As a result, the horizontal hydrodynamic force is:

$$
F=a \int_{0}^{2 \pi} \int_{-d}^{0} \Delta p \cos (\pi-\theta) d \theta d z
$$

Furthermore, using the boundary conditions (3), the free water surface elevations $\xi$ also satisfies that:

$$
\xi_{j}=\operatorname{Re}\left[\frac{i \omega_{j} e^{-i \omega t}}{g}\right] \quad j=1,2 \quad z=0
$$

\section{Convergence studies}

In equation (13) and equation (19), infinite series of the velocity potential are fitted by finite series superposition. The dispersion relationship equation (22) has infinite real roots, corresponding to the non-propagating evanescent mode [9]. Therefore, convergence analysis is necessary for accurate calculation results. For the parameter setting, the wave period uses $T=5 \mathrm{~s}$, and wave height $H=7 \mathrm{~m}$ is applied as the extreme wave height. The water depth $h$ is taken as $100 m$ to simulate the deep sea. The cylinder net cage has a radius $a=50 \mathrm{~m}$ and a length $d=50 \mathrm{~m}$, in which $\gamma=0.05, G=1+0.25 \mathrm{i}, \tau=0.01, \beta=0.1$.

In table 1 , it shows the dimensionless structural deflection amplitudes $|\eta| / d$ with term $N$ from 5 to 30 . When $N$ is greater than 20, the results all converge at the fourth significant digits. Numerically, the term $(n>0)$ generated by the wavenumber of the dispersion relationship equation (22) is relatively small and is only associated with local waves near the cylinder [9]

On the other hand, a relative error $\Delta E_{r}$ is defined as:

$$
\Delta E_{r}=\frac{\left|\sum_{z=0}^{-d} \sum_{\theta=0}^{2 \pi} \Delta \phi(a, \theta, z)\right|_{M}-\left.\sum_{z=0}^{-d} \sum_{\theta=0}^{2 \pi} \Delta \phi(a, \theta, z)\right|_{M=30} \mid}{\left|\sum_{z=0}^{-d} \sum_{\theta=0}^{2 \pi} \Delta \phi(a, \theta, z)\right|_{M=30} \mid}
$$

A plot of $\Delta E_{r}$ with different $M$ from 5 to 30 is shown in figure 2. It can be found that when $M$ is greater than 15 , the relative error with the value of $M=30$ is quite small.

Therefore, in the study, it is sufficient that $N$ is taken 25 , and $M$ is taken 20 for the modelling.

\begin{tabular}{c|c|c|c|c} 
& & $z / d$ & $z / d$ & $z / d$ \\
\hline \multirow{4}{*}{$|\eta| / d\left(\times 10^{-4}\right)$} & $N$ & -0.25 & -0.5 & -0.75 \\
\cline { 2 - 5 } & 5 & 2.353898 & 2.823743 & 1.175003 \\
\cline { 2 - 5 } & 10 & 2.344244 & 2.812012 & 1.171378 \\
\cline { 2 - 5 } & 15 & 2.343985 & 2.811354 & 1.170926 \\
\cline { 2 - 5 } & 20 & 2.343768 & 2.810834 & 1.170588 \\
\cline { 2 - 5 } & 25 & 2.343680 & 2.810628 & 1.170459 \\
\cline { 2 - 5 } & 30 & 2.343594 & 2.810423 & 1.170329 \\
\multicolumn{4}{c}{ Table 1. Convergence studies for term $N$}
\end{tabular}

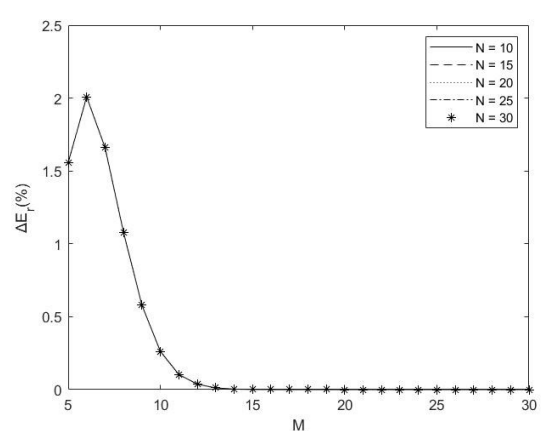

Figure 2. Convergence studies for term $M$

\section{Results and discussion}

A preliminary hydrodynamic analysis for the cylinder fish net cage under the wave loading action has been carried out. The cage is located at the origin of the coordinate, and the waves propagate in the direction of the $x$-axis. The computation 
domain of the water surface uses the range of the radius $R$ is $200 \mathrm{~m}$ and the whole angle range is $2 \pi$. The same parameter settings are adopted as the convergence studies.

A contour plot of the free water surface elevations $\xi$ is shown in figure 3. It can be found that the fishnet cage has obvious perturbations to wave surfaces. The superposition of the incident waves and scattered waves cause the water surface amplitudes to increase in some areas and decrease in some zones. The wave disturbances are mainly concentrated on the cylindrical interior and the lateral sides of the cylinder in the outer region. After the wave passes through the cage, the wave surface gradually restores to its original state.

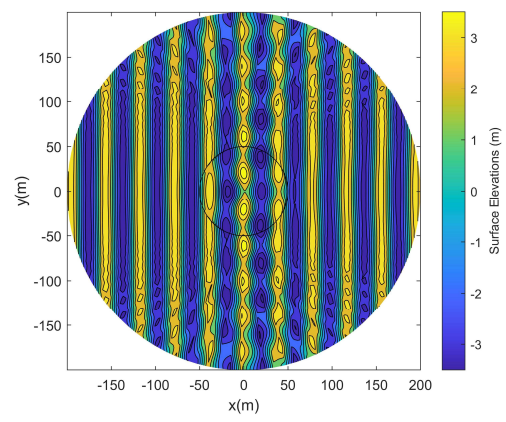

Figure 3. Water surface elevations with $T=5 \mathrm{~s}$ and $H=7 \mathrm{~m}$

In figure 4 , it presents the amplitude distribution of dynamic pressure differences $\Delta p$ on the net surface in front and back. On top of the cylinder, the amplitude of $\Delta p$ is relatively higher than the bottom part values, which indicates the wave effect mainly acts on the upper part of the fish cage. Furthermore, the time series of the horizontal wave hydrodynamic force $F$ is shown in figure 5, where the maximum force is about $6000 \mathrm{kN}$.

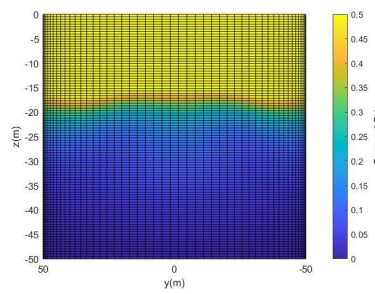

(a)

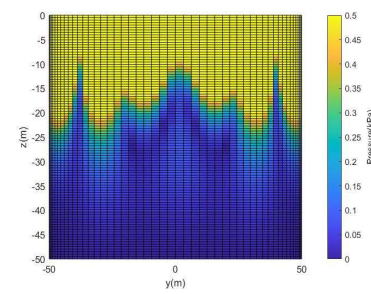

(b)
Figure 4. Hydrodynamic pressure difference amplitude distribution with $T=5 s$ and $H=7 m$ : (a) front, (b) back

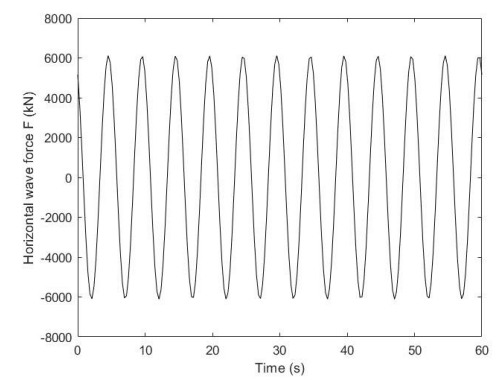

Figure 5. Horizontal wave force $F$ with time series on the cage with $T$ $=5 \mathrm{~s}$ and $\mathrm{H}=7 \mathrm{~m}$

\section{Conclusion}

This paper analyses the wave field and the hydrodynamic forces of the cylindrical net cage using semi-analytical models based on potential flow theory. The net cage is treated as a porous membrane, and the flow-solid boundary conditions satisfy Darcy's law of seepage. The horizontal deflection of the net cage is assumed to satisfy the one-dimensional string vibration equation. In the present model, the fluid is assumed to be inviscid and irrotational, and the velocity potential of the flow field is obtained by solving the Laplace's equation using the Fourier-Bessel expansions method and the least-squares method. Furthermore, the convergence analysis is conducted to determine the appropriate truncation terms in the infinite series.

By calculating the velocity potential, the corresponding velocity and pressure of the flow field can be obtained. From the results of the models, it indicates that the net cage has obvious perturbations to the wave surface. On the other hand, the higher amplitude of the $\Delta p$ is concentrated on the upper part of the cage, but the bottom parts have smaller values. Therefore, the effect of wave actions on the top of the cage is more critical. Besides, the wave forces with the time series acting on the cage are also presented.

This model is applied as an effective tool for hydrodynamic analysis of cylindrical fish net cages in the large-scale domain and deep water. In the future, more complex net cage structures and hydro-elastic behaviour analysis for the net will be studied using this model.

\section{References}

[1] Abul-Azm, A.G. and Williams, A.N., 1987. Interference effects between flexible cylinders in waves. Ocean engineering, 14(1), pp.19-38.

[2] Behera, H. and Sahoo, T., 2015. Hydroelastic analysis of gravity wave interaction with submerged horizontal flexible porous plate. Journal of Fluids and Structures, 54, pp.643-660.

[3] Bi, C.W., Zhao, Y.P., Dong, G.H., Xu, T.J. and Gui, F.K., 2014. Numerical simulation of the interaction between flow and flexible nets. Journal of Fluids and Structures, 45, pp.180-201.

[4] Chwang, A.T., 1983. A porous-wavemaker theory. Journal of Fluid Mechanics, 132, pp.395-406.

[5] Kristiansen, T. and Faltinsen, O.M., 2012. Modelling of current loads on aquaculture net cages. Journal of Fluids and Structures, 34, pp.218-235.

[6] Madsen, O.S., 1974. Wave transmission through porous structures. Journal of the Waterways, Harbors and Coastal Engineering Division, 100(3), pp.169-188.

[7] Su, W., Zhan, J.M. and Huang, H., 2015. Analysis of a porous and flexible cylinder in waves. China Ocean Engineering, 29(3), pp.357-368.

[8] Xu, T.J., Dong, G.H., Zhao, Y.P., Li, Y.C. and Gui, F.K., 2011. Analysis of hydrodynamic behaviors of gravity net cage in irregular waves. Ocean engineering, 38(13), pp.1545-1554.

[9] Zhu, S.P. and Mitchell, L., 2009. Diffraction of ocean waves around a hollow cylindrical shell structure. Wave Motion, 46(1), pp.78-88. 